\title{
A Novel Approach towards Cost Effective Region-Based Group Key Agreement Protocol for Ad Hoc Networks Using Elliptic Curve Cryptography
}

\author{
Krishnan Kumar ${ }^{1}$, J. Nafeesa Begum ${ }^{1}$, V. Sumathy ${ }^{2}$ \\ ${ }^{1}$ Government College of Engineering, Bargur, India \\ ${ }^{2}$ Government College of Technology, Coimbatore, India \\ Email:pkk_kumar@yahoo.com,nafeesa_jeddy@yahoo.com,sumi_gct2001@yahoo.co.in \\ Received January 12, 2010; revised February 19, 2010; accepted March 20, 2010
}

\begin{abstract}
This paper addresses an interesting security problem in wireless ad hoc networks: the dynamic group key agreement key establishment. For secure group communication in an ad hoc network, a group key shared by all group members is required. This group key should be updated when there are membership changes (when the new member joins or current member leaves) in the group. In this paper, we propose a novel, secure, scalable and efficient region-based group key agreement protocol for ad hoc networks. This is implemented by a two-level structure and a new scheme of group key update. The idea is to divide the group into subgroups, each maintaining its subgroup keys using group elliptic curve diffie-hellman (GECDH) Protocol and links with other subgroups in a tree structure using tree-based group elliptic curve diffie-hellman (TGECDH) protocol. By introducing region-based approach, messages and key updates will be limited within subgroup and outer group; hence computation load is distributed to many hosts. Both theoretical analysis and experimental results show that this Region-based key agreement protocol performs well for the key establishment problem in ad hoc network in terms of memory cost, computation cost and communication cost.
\end{abstract}

Keywords: Ad Hoc Network, Region-Based Group Key Agreement Protocol, Elliptic Curve Diffie-Hellman, Tree-Based Group Elliptic Curve Diffie-Hellman

\section{Introduction}

Wireless networks are growing rapidly in recent years. Wireless technology is gaining more and more attention from both academia and industry. Mostly wireless networks are used today e.g. the cell phone networks and the 802.11 wireless LAN, are based on the wireless network model with pre-existing wired network infrastructures. Packets from source wireless hosts are received by near-by base stations, then injected into the underlying network infrastructure and then finally transferred to destination hosts.

Another wireless network model, which is in active research, is the ad-hoc network. This network is formed only by mobile hosts and requires no pre-existing network infrastructure. Hosts with wireless capability form an ad-hoc network, some mobile hosts work as routers to relay packets from source to destination. It is very easy and economic to form an ad-hoc network in real time.
Ad-hoc network is ideal in situations like battlefield or rescuer area where fixed network infrastructure is very hard to deploy.

A mobile ad hoc network is a collection of autonomous nodes that communicate with each other. Mobile nodes come together to form an ad hoc group for secure communication purpose. A key distribution system requires a trusted third party that acts as a mediator between nodes of the network. Ad-hoc networks characteristically do not have a trusted authority. Group key agreement means that multiple parties want to create a common secret key to be used to exchange information securely. Furthermore, group key agreement also needs to address the security issue related to membership changes due to node mobility. The membership change requires frequent changes of group key. This can be done either periodically or updating every membership changes. The changed group key ensures backward and forward secrecy. With frequent changes in group memberships, the 
recent researches began to pay more attention on the efficiency of group key update. Recently, collaborative and group-oriented applicative situations like battlefield, conference room or rescuer area in mobile ad hoc networks have been a current research area. Group key agreement is a building block in secure group communication in ad hoc networks. However, group key agreement for large and dynamic groups in ad hoc networks is a difficult problem because of the requirements of scalability and security under constraints of node available resources and node mobility.

We propose a communication and computation efficient group key agreement protocol in ad-hoc network. In large and high mobility ad hoc networks, it is not possible to use a single group key for the entire network because of the enormous cost of computation and communication in rekeying. So, we divide the group into several subgroups; let each subgroup has its subgroup key shared by all members of the subgroup. Each group has sub group controller node and gateway node, in which the sub group controller node is controller of subgroup and gateway node is controller among subgroups. Let each gateway member contributes a partial key to agree with a common outer group key among the subgroups.

The contribution of this work includes

1) In this paper, we propose a new efficient method for solving the group key management problem in adhoc network. This protocol provides efficient, scalable and reliable key agreement service and is well adaptive to the mobile environment of ad-hoc network.

2) We introduce the idea of subgroup and subgroup key and we uniquely link all the subgroups into a tree structure to form an outer group and outer group key. This design eliminates the centralized key server. Instead, all hosts work in a peer-to-peer fashion to agree on a group key. We use region-based group key agreement (RBGKA) as the name of our protocol. Here we propose a region based group key agreement protocol for ad hoc networks using elliptic curve cryptography called region-based GECDH and TGECDH protocol.

3) We design and implement region-based group key agreement protocol using Java and conduct extensive experiments and theoretical analysis to evaluate the performance like memory cost, communication cost and computation cost of our protocol for Ad-Hoc network.

The rest of the paper is as follows, Section 2 presents the elliptic curve cryptography schemes. Section 3 presents the proposed schemes. Section 4 describes the performance analysis and finally Section 5 concludes the paper.

\section{Elliptic Curve Cryptography}

ECC [1-3] has become the cryptographic choice for ad hoc networks and communication devices due to its size and efficiency benefits. Elliptic curve cipher uses very small keys and is computationally very efficient, which makes it ideal for the smaller, less powerful devices being used today by majority of individuals to access network services. The elliptic curve cryptosystem (ECCS) is a crypto-algorithm method of utilizing a discrete logarithm problem (DLP) over the points on an elliptic curve.

An elliptic curve is usually defined over two finite fields: the prime finite field $F_{p}$ containing $p$ elements and the characteristic finite field containing $2^{m}$ elements. This paper focuses on the prime finite field. Let $F_{p}$ be a prime finite field, $p$ is an odd prime number, and $a, b \varepsilon F_{p}$ satisfy $4 a^{3}+27 b^{2} \neq 0(\bmod p)$, then an elliptic curve $E\left(F_{p}\right)$ over $F_{p}$ defined by the parameters $a, b \varepsilon F_{p}$ consists of the set of solutions or points $P=(x, y)$ for $x, y \varepsilon F_{p}$ to the equation:

$$
y^{2}=x^{3}+a x+b(\bmod p)
$$

The equation $y^{2}=x^{3}+a x+b(\bmod p)$ is called the defining equation of $E\left(F_{p}\right)$. For a given point $P=\left(x_{P}, y_{P}\right), x_{P}$ is called the $x$-coordinate of $P$, and $y_{P}$ is called the $y$-coordinate of $P$.

Cryptographic schemes based on ECC rely on scalar multiplication of elliptic curve points. Given an integer $k$ and a point $P \varepsilon E\left(F_{p}\right)$, scalar multiplication is the process of adding $P$ to itself $k$ times. The result of this scalar multiplication is denoted $k^{*} P$ or $k P$. Scalar multiplication of elliptic curve points can be computed efficiently using the addition rule together with the double-and-add algorithm or one of its variants.

\subsection{Two Parties Elliptic Curve Diffie-Hellman Protocol}

Similar to DLP-based Diffie-Hellman key exchange agreement, a key exchange between users $A$ and $B$ using elliptic curve Diffie-Hellman (ECDH) $[2,3]$ can be accomplished as follows:

1) $A$ selects an integer $n_{A}$ less than $p$, this is $A$ 's private key. $A$ then generates a public key $P_{A}=n_{A} * G$; the public key is a point in $\operatorname{Ep}(a, b)$.

2) $B$ similarly selects a private key $n_{B}$ and computes a public key $P_{B}=n_{B} * G$.

3) $A$ and $B$ generates the secret key $K=n_{A} * P_{B}, K=n_{B}$ $* P_{A}$ respectively.

The two calculations in step 3 produce the same result because

$$
n_{A} * P_{B}=n_{A} *\left(n_{B} * G\right)=n_{B} *\left(n_{A} * G\right)=n_{B} * P_{A} .
$$

The secret key $K$ is a point in the elliptic curve. 


\section{Proposed Scheme}

\subsection{Motivation}

There has been a growing demand in the past few years for security in collaborative environments deployed for emergency services where our approach can be carried out very efficiently are shown in Figure 1. Confidentiality becomes one of the top concerns to protect group communication data against passive and active adversaries. To satisfy this requirement, a common and efficient solution is to deploy a group key shared by all group application participants. Whenever a member leaves or joins the group, or whenever a node failure or restoration occurs, the group key should be updated to provide forward and backward secrecy. Therefore, a key management protocol that computes the group key and forwards the rekeying messages to all legitimate group members is central to the security of the group application.

In many secure group applications [4,5], a Region based contributory GKA schemes may be required. In such cases, the group key management should be both efficient and fault-tolerant. In this paper, we describe a military scenario (Figure 2). A collection of wireless mobile devices are carried by soldiers or battlefield tanks. These mobile devices cooperate in relaying packets to dynamically establish routes among themselves to and processing

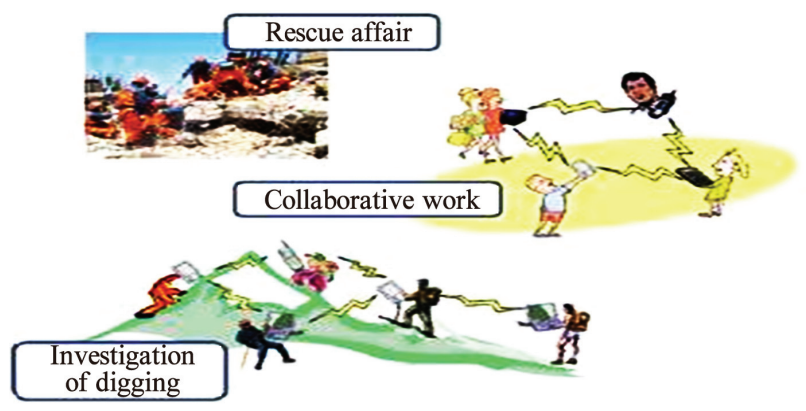

Figure 1. Secure group applications.

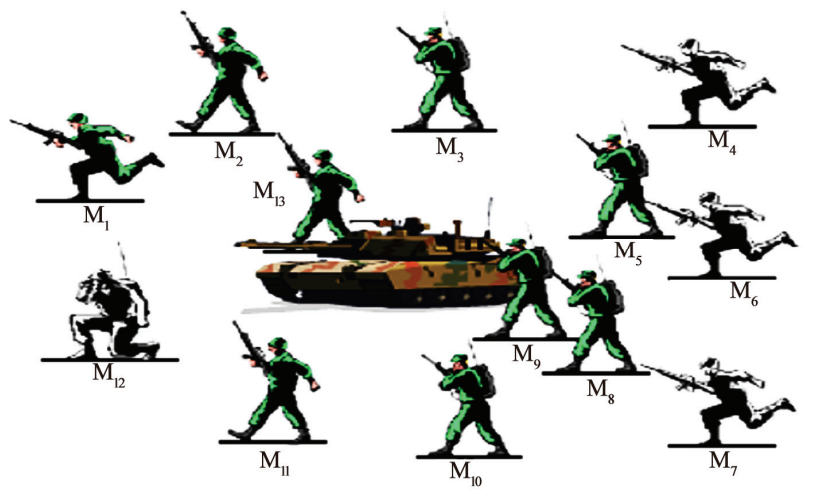

Figure 2. Battlefield scenario. form their own network "on the fly". However, all nodes except the one with the tank, have limited battery power capacities. For the sake of power-consumption and computational efficiency, the tank can work as the Gateway member while a contributed group key management scheme is deployed.

\subsection{System Model}

\subsubsection{Overview of Region-Based Group Key Agreement Protocol}

The goal of this paper is to propose a communication and computation efficient group key establishment protocol in ad-hoc network. The idea is to divide the multicast group into several subgroups, let each subgroup has its subgroup key shared by all members of the subgroup. Each Subgroup has subgroup controller node and a gateway node, in which Subgroup controller node is the controller of subgroup and a Gateway node is controller of subgroups controller.

For example, in Figure 3, all member nodes are divided into number of subgroups and all subgroups are linked in a tree structure as shown in Figure 4.

One of the members in the subgroup is subgroup controller. The last member joining the group acts as a subgroup controller. Each outer group is headed by the outer group controller. In each group, the member with high processing power, memory, and Battery power acts as a

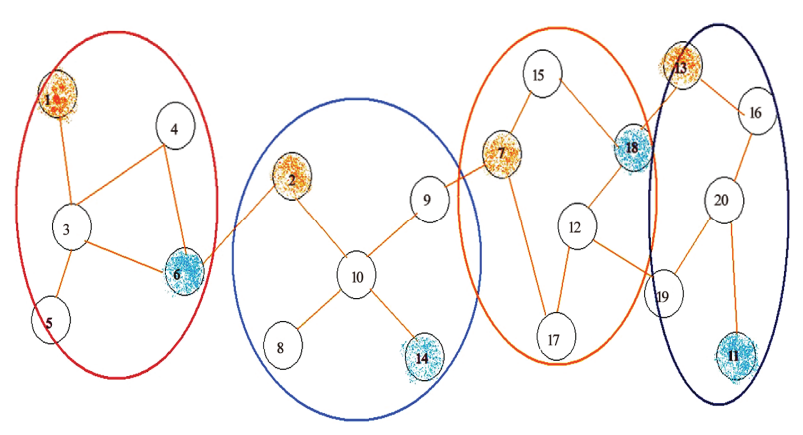

Figure 3. Members of group are divided into subgroups.

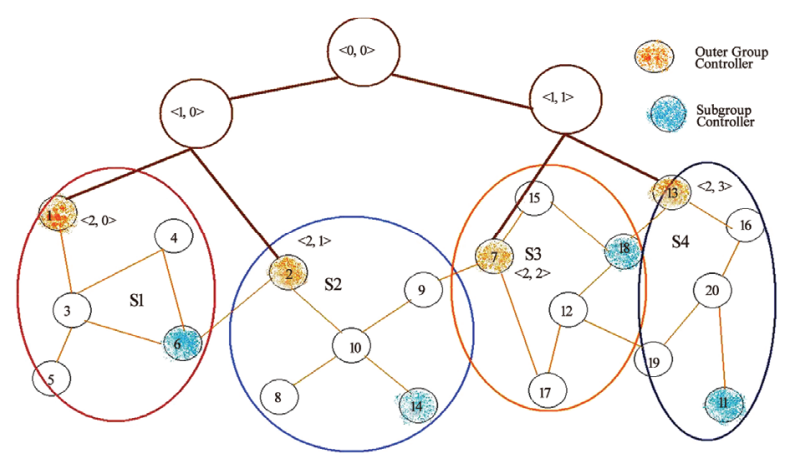

Figure 4. Subgroups link in a tree structure. 
gateway member. Outer group messages are broadcast through the outer group and secured by the outer group key while subgroup messages are broadcast within the subgroup and secured by subgroup key.

Let $N$ be the total number of group members, and $M$ be the number of the subgroups in each subgroup, then there will be $N / M$ subgroups, assuming that each subgroup has the same number of members.

There are two shared keys in the Region-Based Group Key Agreement Scheme:

1) Outer Group Key $(\mathrm{KG})$ is used to encrypt and decrypt the messages broadcast among the subgroup controllers.

2) The Subgroup Key (KR) is used to encrypt and decrypt the Sub Group level messages broadcast to all sub group members.

In our Region-Based Key Agreement protocol shown in Figure 5. a Subgroup Controller communicates with the member in the same region using a Regional key (i.e. Subgroup key ) KR. The Outer Group key KG is derived from the Outer Group Controller. The Outer Group Key $\mathrm{KG}$ is used for secure data communication among subgroup members. These two keys are rekeyed for secure group communications depending on events that occur in the system. The layout of the network is as shown in below Figure 5.

Assume that there are total $N$ members in Secure Group Communication. After sub grouping process (algorithm 1), there are $S$ subgroups $M_{1}, M_{2} \ldots M_{s}$ with $n_{1}$, $n_{2} \ldots n_{s}$ members.

\footnotetext{
Algorithm 1. Region-Based Key Agreement Protocol

1) The Subgroup Formation

The number of members in each subgroup is

$$
\mathbf{N} / \mathbf{S}<\mathbf{1 0 0} \text {. }
$$

where,

$\mathrm{N}$ - is the group size. and

$\mathrm{S}-$ is the number of subgroups.

Assuming that each subgroup has the same number of members.

2) The Contributory Key Agreement protocol is implemented among the group members. It consists of three stages.

a. To find the Subgroup Controller for each subgroups.

b. GECDH protocol is used to generate one common key for each subgroup headed by the subgroup controller.

c. Each subgroup gateway member contributes partial keys to generate a one common backbone key (i.e. Outer group Key (KG)) headed by the Outer Group Controller using TGECDH protocol.

3) Each Group Controller (sub /Outer) distributes the computed public key to all its members.
}

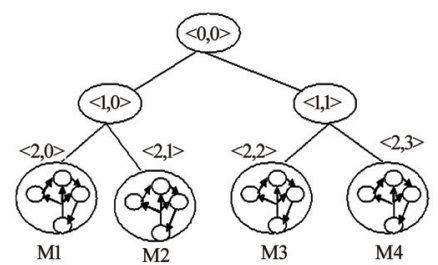

Figure 5. Region based group key agreement.

A Regional key KR is used for communication between a subgroup controller and the members in the same region. The Regional key KR is rekeyed whenever there is a membership change event and subgroup joins/ leaves and member failure. The Outer Group key KG is rekeyed whenever there is a join/leave subgroup controllers and member failure to preserve secrecy.

The members within a subgroup use Group Elliptic Curve Diffie-Hellman (GECDH) Contributory Key Agreement. Each member within a subgroup contributes his share in arriving at the subgroup key. Whenever membership changes occur, the subgroup controller or previous member initiates the rekeying operation.

The gateway member initiates communication with the neighboring member belonging to another subgroup and mutually agree on a key using Tree-Based Group Elliptic Curve Diffie-Hellman (TGECDH) Contributory Key Agreement protocol to be used for inter subgroup communication between the two subgroups. Any member belonging to one subgroup can communicate with any other member in another subgroup through this member as the intermediary. In this way adjacent subgroups agree on outer group key. Whenever membership changes occur, the outer group controller or previous group controller initiates the rekeying operation.

Here, we prefer the subgroup key to be different from the key for backbone. This difference adds more freedom of managing the dynamic group membership. In addition by using this approach can potentially save the communication and computational cost.

\subsection{Network Dynamics}

The network is dynamic in nature. Many members may join or leave the group. In such case, a group key management system should ensure that backward and forward secrecy is preserved.

\subsubsection{Member Join}

When a new member joins, it initiates communication with the subgroup controller. After initialization, the subgroup controller changes its contribution and sends public key to this new member. The new member receives the public key and acts as a group controller by initiating the rekeying operations for generating a new key for the subgroup. The rekeying operation is as follows. 
New node $\frac{\text { Join request }}{\text { Subgroup Controller }} \rightarrow$ change its contribution and send public key to
$\rightarrow$ New Node

New Node $\stackrel{\text { Acts as }}{\rightarrow}$ New Subgroup Controller

puts its contribution to all the public key value \&

New Subgroup Controller $\frac{\text { Multicast this public key value to }}{\rightarrow} \rightarrow$ the entire member in the subgroup

Each Member $\frac{\text { put is contribution to the public value \& Compute }}{\rightarrow}$ New Subgroup Key

\subsubsection{Member Leave}

\subsubsection{When a Subgroup member Leaves}

When a member leaves the Subgroup Key of the subgroup to which it belongs must be changed to preserve the forward secrecy. The leaving member informs the subgroup controller. The subgroup controller changes its private key value, computes the public value and broadcasts the public value to all the remaining members. Each member performs rekeying by putting its contribution to public value and computes the new Subgroup Key. The rekeying operation is as follows.

$$
\text { Leaving Node } \stackrel{\text { Leaving Message }}{\rightarrow} \text { Subgroup Controller }
$$

changes its private key value, compute the public key value and

$$
\text { Subgroup Controller } \longrightarrow \text { Multicast the public key value to } \rightarrow \text { All the remaining Member }
$$

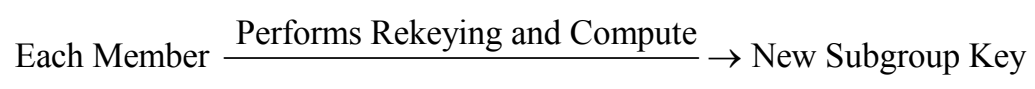

\subsubsection{When Subgroup Controller Leaves}

When the Subgroup Controller leaves, the Subgroup key used for communication among the subgroup controller needs to be changed. This Subgroup Controller informs the previous Subgroup Controller about its desire to leave the subgroup which initiates the rekeying procedure. The previous subgroup controller now acts as a Subgroup controller. This Subgroup controller changes its private contribution value and computes all the public key values and broadcasts to all the remaining members of the group. All subgroup members perform the rekeying operation and compute the new subgroup key. The rekeying operation is as follows.

$$
\text { Leaving Subgroup Controller } \frac{\text { Leaving Message }}{\rightarrow} \rightarrow \text { Old Subgroup Controller }
$$

change its private value,compute the all

$$
\begin{aligned}
\text { Old Subgroup Controller } \frac{\text { public key value and Multicast }}{\text { Subgroup Member } \frac{\text { Perform Rekeying and Compute }}{}} \rightarrow \text { Remaining Member in the group } \\
\rightarrow \text { New Subroup Key }
\end{aligned}
$$

\subsubsection{When Outer Group Controller Leaves}

When the Outer group Controller leaves, the Outer group key used for communication among the Outer group need to be changed. This Outer group Controller informs the previous Outer group Controller about its desire to leave the Outer group which initiates the rekeying procedure. The previous Outer Group controller now be- comes the New Outer group controller. This Outer group controller changes its private contribution value and computes the public key value and broadcast to the entire remaining member in the group. All Outer group members perform the rekeying operation and compute the new Outer group key. The rekeying operation is as follows.

$$
\text { Leaving Outer group Controller } \stackrel{\text { Leaving Message }}{\rightarrow} \text { Old Outer group Controller }
$$

change its private value,compute the all

Old Outer group Controller $\frac{\text { public key value and Multicast }}{\longrightarrow} \rightarrow$ Remaing Member in the Outer group 


$$
\text { Outer group Member } \stackrel{\text { Perform Rekeying and Compute }}{\rightarrow} \text { New Outer group Key }
$$

\subsubsection{When Gateway Member Leaves}

When a gateway member leaves the subgroup, it delegates the role of the gateway to the adjacent member having high processing power, memory, and Battery power and acts as a new gateway member. Whenever the gateway member leaves, all the two keys should be changed. These are

i. Outer group key among the subgroup.

ii. Subgroup key within the subgroup.

In this case, the subgroup controller and outer group controller perform the rekeying operation. Both the Controller leaves the member and a new gateway member is selected in the subgroup, performs rekeying in the subgroup. After that, it joins in the outer group. The procedure is same as joining the member in the outer group.

\subsection{Communication Protocol}

The members within the subgroup have communication using subgroup key. The communication among the subgroup members takes place through the gateway member.

\subsubsection{Communication within the Subgroup}

The sender member encrypts the message with the subgroup key (KR) and multicasts it to all member in the subgroup. The subgroup members receive the encrypted message, perform the decryption using the subgroup key (KR) and get the original message. The communication operation is as follows.

$$
\begin{gathered}
\text { Source Member } \frac{\mathrm{E}_{\mathrm{KR}}[\text { Message }] \& \text { Multicast }}{} \rightarrow \text { Destination Member } \\
\text { Destination Member } \frac{\mathrm{D}_{\mathrm{KR}}\left[\mathrm{E}_{\mathrm{KR}}[\text { Message }]\right]}{\rightarrow \text { Original Message }}
\end{gathered}
$$

\subsubsection{Communication among the Subgroup}

The sender member encrypts the message with the subgroup key (KR) and multicasts it to all members in the subgroup. One of the members in the subgroup acts as a gate way member. This gateway member decrypts the message with subgroup key and encrypts with the outer group key $(\mathrm{KG})$ and multicast to the entire gateway member among the subgroup. The destination gateway member first decrypts the message with outer group key. Then encrypts with subgroup key and multicast it to all members in the subgroup. Each member in the subgroup receives the encrypted message and performs the decryption using subgroup key and gets the original message. In this way the region-based group key agreement protocol performs the communication. The communication operation is as follows.

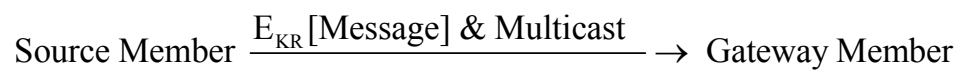

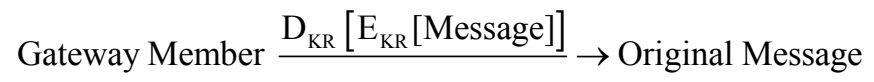

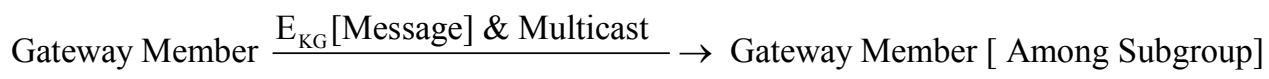

$$
\begin{aligned}
& \text { Gateway Member } \frac{\mathrm{D}_{\mathrm{KG}}\left[\mathrm{E}_{\mathrm{KG}}[\text { Message }]\right]}{\rightarrow \text { Original Message }} \\
& \text { Gateway Member } \frac{\mathrm{E}_{\mathrm{KR}}[\text { Message }] \& \text { Multicast }}{\rightarrow} \text { Destination Member }
\end{aligned}
$$

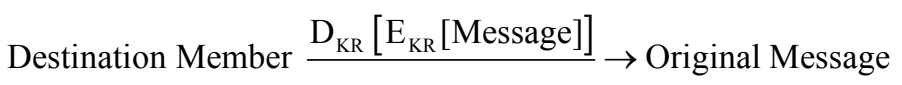

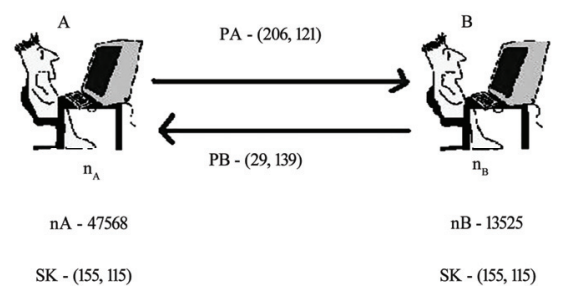

\subsection{Applying Elliptic Curve Based Diffie-Hellman Key Exchange}

\subsubsection{Member Join}

User A and user B are going to exchange their keys (Figure 6): Take $p=211, E p=(0,-4)$, which is equivalent to the curve $y^{2}=x^{3}-4$ and $G=(2,2)$. A's private key is $n A$ $=47568$, so A's public key PA $=47568(2,2)=(206,121)$,
Figure 6. User-A and User -B join the group. 
B's private key is $\mathrm{nB}=13525$, so B's public key $\mathrm{PB}=$ $13525(2,2)=(29,139)$. The group key is computed (Figure 6) as User A sends its public key $(206,121)$ to user B, then user B computes their Subgroup key as nB (A's Public key $)=13525(206,121)=(\mathbf{1 5 5}, \mathbf{1 1 5})$. User B sends its public key $(29,139)$ to User A, then User A compute their Subgroup key as nA (B's Public key) = $47568(29,139)=(\mathbf{1 2 0}, \mathbf{1 8 0})$

When User $\mathrm{C}$ is going to join in the group, C's private key becomes $\mathrm{nC}=82910$. Now, User C becomes a Subgroup Controller. Then, the key updating process will begin as follows: The previous Subgroup Controller User B sends the intermediate key as (B's Public key \$ A's Public Key $\$$ Group key of A\&B) $=((29,139) \$(206,121)$ $\$(155,115))$ User $C$ separates the intermediate key as B's Public key, A's Public Key and Group key of A\&B $=(29,139),(206,121)$ and $(155,115)$. Then, User C generates the new Subgroup key as $\mathrm{nC}$ (Subgroup key of $\mathrm{A} \& \mathrm{~B})=82910(155,115)=(\mathbf{1 2 0 , 3 1})$. Then, User $\mathrm{C}$ broadcasts the intermediate key to User A and User B. That intermediate key is ((Public key of B \& C) \$ (Public key of A \& C $)=((131,84) \$(147,97))$. Now, User B extracts the value of public key of $A \& C$ from the value sent by User C. Then User B compute the new Subgroup key as follows: $\mathrm{nB}$ (Public key of A\&C) $=13525(147,97)$ $=(\mathbf{1 2 0}, \mathbf{3 1})$. Similarly, User A extracts the value of public key of B \& C from intermediate key, sent by User C. Then User A compute the new Subgroup key as follows: $\mathrm{nA}($ public key of $\mathrm{B} \& \mathrm{C})=47568(131,84)=(\mathbf{1 2 0}, \mathbf{3 1})$. Therefore, New Subgroup Key of A, B and C $=(\mathbf{1 2 0}, \mathbf{3 1})$ as shown in the Figure 7.

The same procedure is followed when User D joins as shown in the Figure 8.

\subsubsection{Member Leave}

When a user leaves (Figure 9) from the Subgroup, then the Subgroup controller changes its private key. After that, it broadcasts its new public key value to all users in the Subgroup. Then, new Subgroup key will be generated. Let us consider, User B is going to leave, then the Subgroup Controller D changes its private key $\mathrm{nD}^{\prime}=$ 43297, so public key of User A \& User C $=(198,139)$ $\$(136,11)$. Then the new Subgroup Key generated is = $43297(198,139)=(\mathbf{2 0 7}, \mathbf{1 1 5})$. Then, User A \& User C

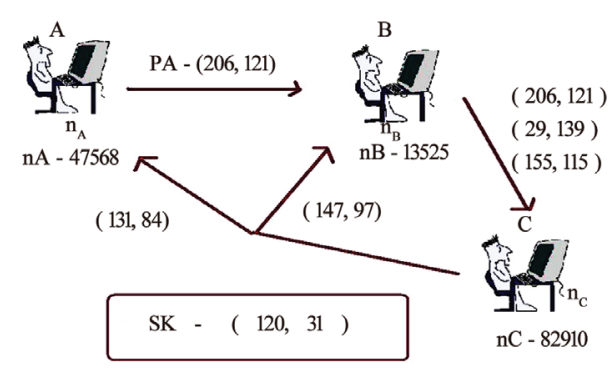

Figure 7. User - $C$ joins in the group.

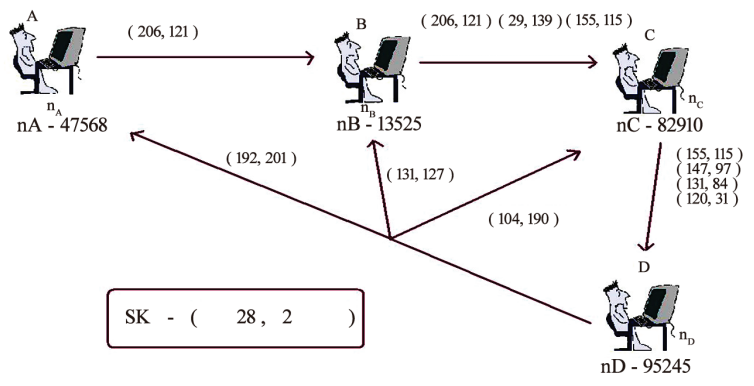

Figure 8. User-D joins in the group.

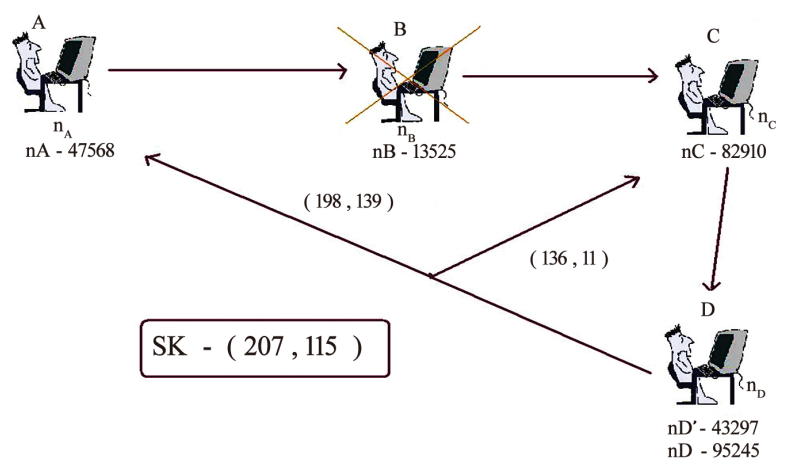

Figure 9. User-B leaves from the group.

computes the new Subgroup Key by using new public key. Therefore, the new Subgroup Key is $(\mathbf{2 0 7}, \mathbf{1 1 5})$.

\subsubsection{Group Controller Leave}

When a Subgroup controller leaves (Figure 10) from the group, then the previous Subgroup controller changes its private key. After that, it broadcasts its new public key value to all users in the group. Then, new Subgroup key will be generated. Let us consider, Subgroup Controller User D going to leave, then the previous Subgroup controller User C act as Subgroup Controller and changes its private key $\mathrm{nC}^{\prime}=52898$, and computes the public key of B\&C $\$ A \& C=(16,111) \$(181,2)$. Then the new Subgroup Key generated is $=52898(21,103)=(\mathbf{1 9 8}, 139)$. Then, User A \& User B compute the new Subgroup Key by using new public key. Therefore, the new Subgroup Key is $(\mathbf{1 9 8 , 1 3 9 )}$.

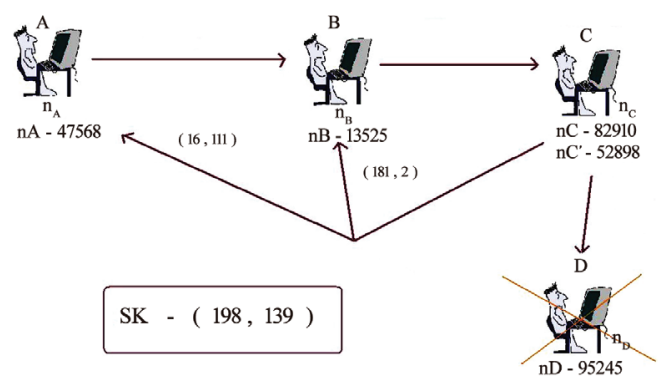

Figure 10. Group controller leaves from the group. 


\subsection{Tree-Based Group Elliptic Curve Diffie-Hellman Protocol}

The proposed protocol (Figure 11), Tree-based group Elliptic Curve Diffie-Hellman (TGECDH), is a variant of TGDH based on ECDLP. In TGECDH, a binary tree is used to organize group members. The nodes are denoted as $<$,, $\mathrm{v}>$, where $0<=\mathrm{v}<=21$. -1 since each level 1 hosts at most 21. nodes. Each node $<1, \mathrm{v}>$ is associated with the key $\mathrm{K}<\mathrm{l}, \mathrm{v}>$ and the blinded key $\mathrm{BK}<\mathrm{l}, \mathrm{v}>=\mathrm{F}(\mathrm{K}<\mathrm{l}, \mathrm{v}>)$ where the function $\mathrm{F}()$ is scalar multiplication of elliptic curve points in prime field. Assuming a leaf node $<1, \mathrm{v}>$ hosts the member Mi, the node $<1, v>$ has Mi's session random key $\mathrm{K}<\mathrm{l}, \mathrm{v}>$. Furthermore, the member $\mathrm{Mi}$ at node $<$ l. $\mathrm{v}>$ knows every key in the key-path from $<1, \mathrm{v}>$ to $<0,0>$. Every key $\mathrm{K}<1, \mathrm{v}>$ is computed recursively as follows:

$$
\begin{aligned}
K_{<l, v>} & =K_{<l+1,2 v>} B K_{<l+1,2 v+1>} \bmod p \\
& =K_{<l+1,2 v+1>} B K_{<l+1,2 v>} \bmod p \\
& =K_{<l+1,2 v>} K_{<l+1,2 v+1>} G \bmod p \\
& =F\left(K_{<l+1,2 v>} K_{<l+1,2 v+1>}\right)
\end{aligned}
$$

It is not necessary for the blind key $\mathrm{BK}<\mathrm{l,v}>$ of each node to be reversible. Thus, simply use the $\mathrm{X}$-coordinate of $\mathrm{K}<\mathrm{l}, \mathrm{v}>$ as the blind key. The group session key can be derived from $\mathrm{K}<0,0>$. Each time when there is member join/leave, the outer group controller node calculates the group session key first and then broadcasts the new blind keys to the entire group and finally the remaining group members can generate the group session key.

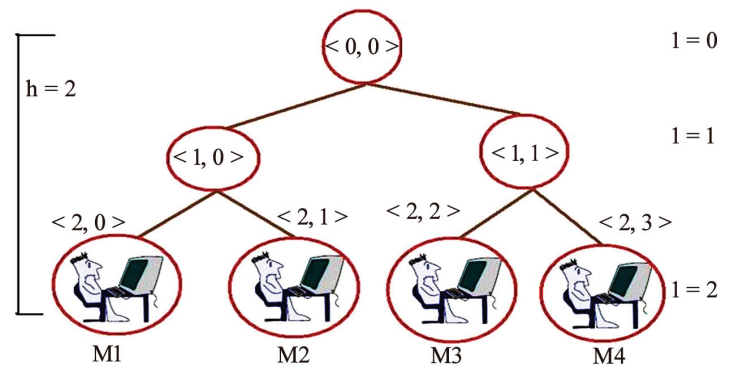

Figure 11. Key tree.

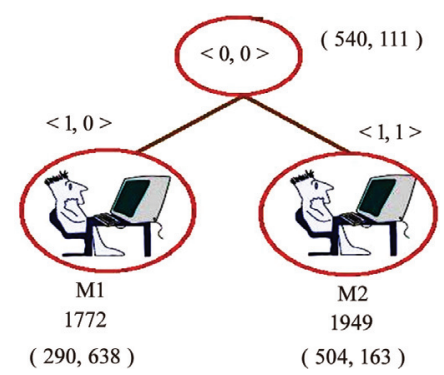

Figure 12. User $M_{1}$ and $M_{2}$ join the group.

\subsubsection{When Node $M_{1} \& M_{2}$ Join the Group}

User $\mathrm{M}_{1}$ and User $\mathrm{M}_{2}$ are going to exchange their keys: Take $\mathbf{p}=\mathbf{7 5 1}, \mathbf{E p}=\mathbf{( 1 , 1 8 8 )}$, which is equivalent to the curve $\mathbf{y}^{2}=\mathbf{x}^{3}+\mathbf{x}+\mathbf{1 8 8}$ and $\mathbf{G}=\left(\mathbf{0 , 3 7 6 )}\right.$. User $\mathrm{M}_{1}$ 's private key is $\mathbf{1 7 7 2}$, so $\mathrm{M}_{1}$ 's public key is $(\mathbf{2 9 0 , 6 3 8 )}$. User $\mathrm{M}_{2}$ 's private key is 1949, so $\mathrm{M}_{2}$ 's public key is $(504,163)$. The Outer Group key is computed (Figure 12) as User $\mathrm{M}_{1}$ sends its public key $\left(\mathbf{2 9 0 , 6 3 8 )}\right.$ to user $\mathrm{M}_{2}$, the User $\mathrm{M}_{2}$ computes their group key as $\mathrm{PV}(0,0)=\mathrm{X}_{\mathrm{co}}(\mathrm{PV}(1,0)$ $* \mathrm{~PB}(1,1))$ and $\mathrm{PB}(0,0)=\mathrm{PV}(0,0) * \mathrm{G}=(\mathbf{5 4 0}, \mathbf{1 1 1})$. Similarly, User $\mathrm{M}_{2}$ sends its public key $\left(\mathbf{5 0 4 , 1 6 3 )}\right.$ to user $\mathrm{M}_{1}$, and then the user $\mathrm{M}_{1}$ computes their group key as $\mathbf{( 5 4 0 , 1 1 1 )}$. Here, Outer Group controller is User $\mathrm{M}_{2}$.

\subsubsection{When $3^{\text {rd }}$ Node Join}

When User $\mathrm{M}_{3}$ joins the group, the old Outer group controller $\mathrm{M}_{2}$ changes its private key value from 1949 to 2835 and passes the public key value and tree to User $\mathrm{M}_{3}$. Now, $\mathrm{M}_{3}$ becomes new Outer group controller. Then, $\mathrm{M}_{3}$ generates the public key $(623,52)$ from its private key as 14755 and computes the Outer group key as (664,736) shown in Figure 13. $\mathrm{M}_{3}$ sends Tree and public key to all users. Now, user $\mathrm{M}_{1}$ and $\mathrm{M}_{2}$ compute their group key. The same procedure is followed by joining the User $\mathrm{M}_{4}$ as shown in Figure 14.

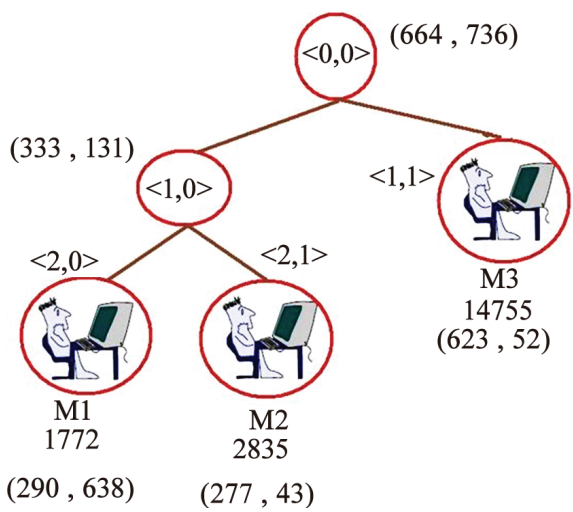

Figure 13. User $M_{3}$ joins the group.

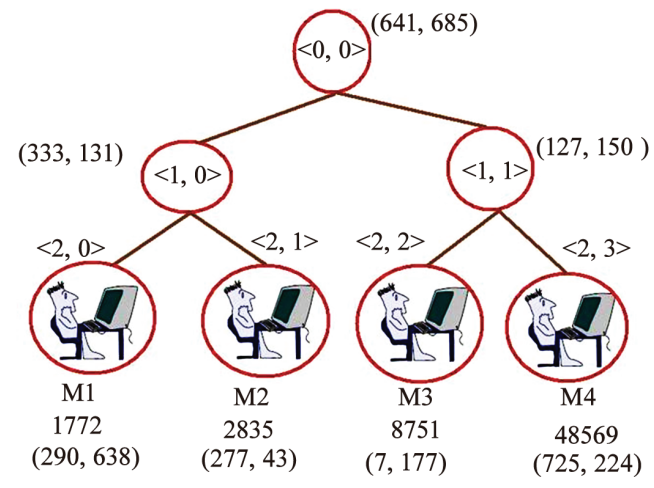

Figure 14. User $M_{4}$ joins the group. 


\subsubsection{Leave Protocol}

There are two types of leave, 1) Gateway Member Leave and 2) Outer Group Controller Leave.

\subsubsection{Gateway Member Leave}

When user $\mathrm{M}_{3}$ leaves (Figure 15) the Outer group, then the Group controller changes its private key 48569 to 98418 and outer group key is recalculated as $(\mathbf{4 2 8 , 6 8 6 )}$. After that, it broadcasts its Tree and public key value to all users in the Outer group. Then, the new Outer group key will be generated by the remaining users.

\subsubsection{When an Outer Group Controller Leaves}

When an Outer Group Controller Leaves (Figure 16) from the group, then its sibling act as a New Outer Group Controller and changes its private key value 8751 to 19478 and recalculates the group key as $(681,475)$. After that, it broadcast its Tree and public key value to all users in the Outer group. Then, the new Outer group key will be generated by the remaining users.

\section{Performance Analysis}

The performance of the proposed scheme is analyzed in terms of the storage overhead, communication overhead and the computation overhead. The storage overhead

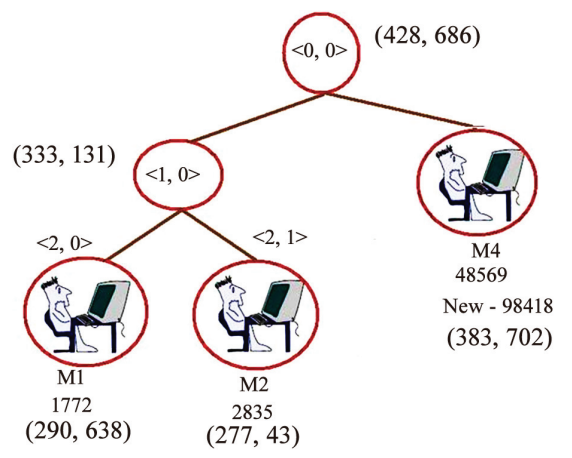

Figure 15. User $M_{3}$ leaves from the group.

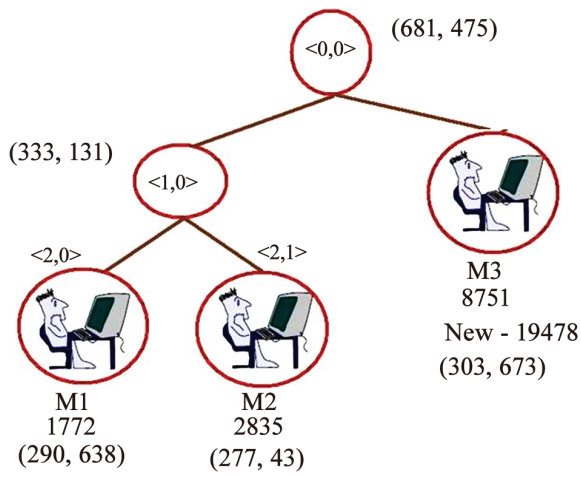

Figure 16. Outer group controller leaves from the group. represents the storage capacity needed for storing the key information. The communication overhead corresponds to the rekeying messages that are transmitted for group communication. The computation overhead is the computation involved in maintaining membership changes.

\subsection{Storage Overhead}

Storage overhead can be considered as the memory capacity required for maintaining the keys, which is directly proportional to the number of members if the key size are same. In this section, the storage cost is formulated, both at gateway member and at each member. Thus our approach consumes very less memory when compared to TGDH and GDH. TGDH and GDH occupy large memory when members go on increasing. But our Region-based Approach takes very less memory even when the members get increased. Consider (Table 1 and Figure 17) there are 1024 members in a group our Region-based approach consumes $10 \%$ of memory when compared to GDH and 5\% when compared to TGDH. The ratio of memory occupied is very less in our approach.

Table 1. Memory cost.

\begin{tabular}{|c|c|c|c|}
\hline \multicolumn{2}{|c|}{ Protocol } & \multirow{2}{*}{$\begin{array}{c}\text { Keys } \\
2 \\
\end{array}$} & \multirow{2}{*}{$\begin{array}{c}\text { Public } \\
N+1\end{array}$} \\
\hline $\mathrm{GDH}$ & Concretely & & \\
\hline \multirow{2}{*}{ TGDH } & $\operatorname{Per}(\mathrm{L}, \mathrm{V})$ & $L+1$ & $2 N-2$ \\
\hline & Averagely & {$\left[\log _{2} N\right]+1$} & $2 N-2$ \\
\hline \multirow{2}{*}{$\begin{array}{c}\text { Region Based } \\
\text { Protocol } \\
\text { (GECDH } \\
\text { \&TGECDH) }\end{array}$} & $\begin{array}{l}\text { Member/Subgroup } \\
\text { Controller }\end{array}$ & 2 & $X+1$ \\
\hline & $\begin{array}{l}\text { Gateway } \\
\text { Member/Outer } \\
\text { group Controller }\end{array}$ & $2+M$ & $X+2 Y-1$ \\
\hline
\end{tabular}

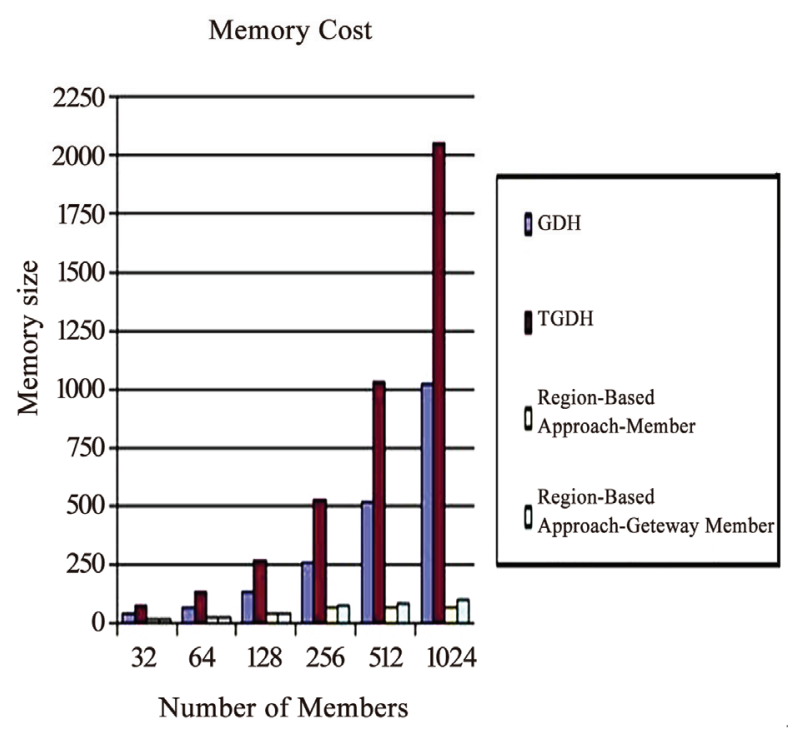

Figure 17. Memory cost. 


\subsection{Communication Overhead}

GDH is the most expensive protocol. TGDH consumes more bandwidth. The communication and computation of TGDH depends on trees height, balance of key tree, location of joining tree, and leaving nodes. Hence GDH has more communication efficiency than TGDH protocol. But our approach depends on the number of members in the subgroup, number of Group Controllers, and height of tree. So the amount spent on communication is very much less when compared to GDH and TGDH.

Consider (Table 2 \& Figures 18 \& 19) there are 512 members in a group our approach consumes only $10 \%$ of Bandwidth when compared to GDH and TGDH. So our approach consumes low Bandwidth.

Table 2. Communication cost.

\begin{tabular}{|c|c|c|c|c|c|}
\hline \multirow{2}{*}{\multicolumn{2}{|c|}{ Protocol Suite }} & \multirow[b]{2}{*}{ Protocol } & \multirow[b]{2}{*}{ Rounds } & \multicolumn{2}{|c|}{ Communication Cost } \\
\hline & & & & $\begin{array}{l}\text { Unicast } \\
\text { Size }\end{array}$ & $\begin{array}{l}\text { Broadcast } \\
\text { Size }\end{array}$ \\
\hline \multirow{2}{*}{\multicolumn{2}{|c|}{ GDH }} & Join & 2 & $N+1$ & $N+1$ \\
\hline & & Leave & 1 & 0 & $N-1$ \\
\hline \multirow{2}{*}{\multicolumn{2}{|c|}{ TGDH }} & Join & 2 & 0 & $2 N+2$ \\
\hline & & Leave & 1 & 0 & $2 N-4$ \\
\hline \multirow{4}{*}{ 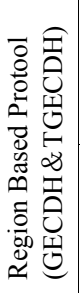 } & 产言 & Join & 2 & $X+1$ & $X+1$ \\
\hline & 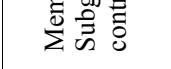 & Leave & 1 & 0 & $X-1$ \\
\hline & $\overrightarrow{\vec{d}}$ & Join & 2 & $X+1$ & $X+2 Y+3$ \\
\hline & 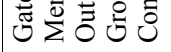 & Leave & 1 & 0 & $X+2 Y-5$ \\
\hline
\end{tabular}

\section{Communication cost-Join}

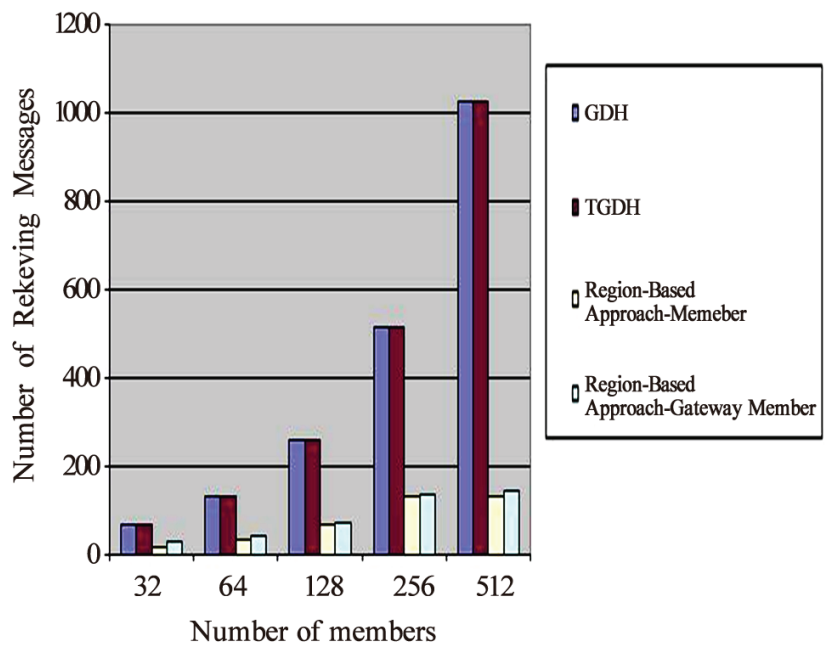

Figure 18. Communication cost - join.
Communication cost-Leave

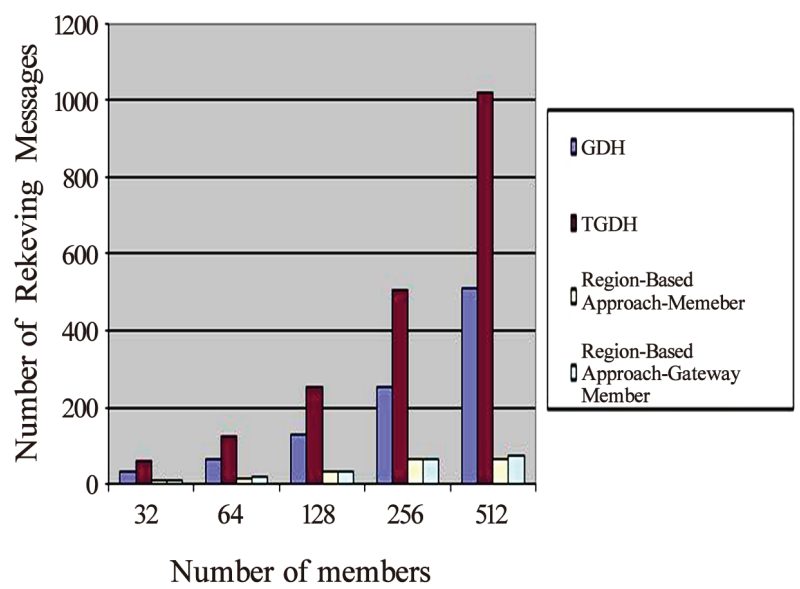

Figure 19. Communication cost - leave.

\subsection{Computation Overhead}

The Figure 20 shows that GECDH and TGECDH schemes have lower computation time than Group Diffie-Hellman (GDH) schemes for member join operations. The computation time of GDH is $\mathbf{2 . 2}$ times that of GECDH and TGDH is $\mathbf{1 . 7}$ times that of TGE-CDH on average for member join operations. The computation time for member leave operations of TGE-CDH schemes are far better than group Diffie-Hellman schemes for member leave operations as shown in the Figure 21. Performance wise our approach leads the GDH \& TGDH methods, even for the very large groups.

The performance of GECDH \& TGECDH over wireless ad hoc Networks can be summarized as follows:

1) It uses smaller keys.

2) It uses less computation time than the DLP-based scheme for the same security level.

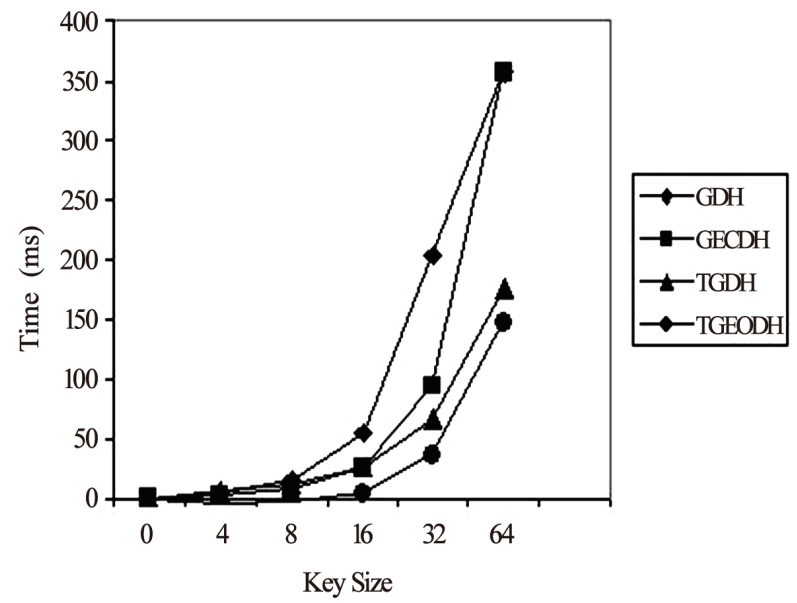

Figure 20. Computation time for member join. 


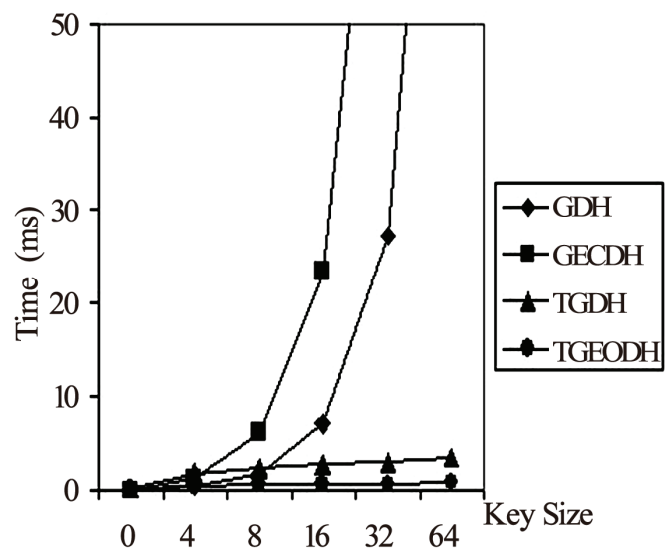

Figure 21. Computation time for member leave.

3) Smaller packets are used to handle high bit error rate Wireless links.

\section{Conclusions}

In this paper, a region-based key agreement scheme has been proposed and implemented, which can enhance the secure group communication performance by using multiple group keys. In contrast to other existing schemes using only single key, the new proposed scheme exploits asymmetric key, i.e. an outer group key and multiple Subgroup keys, which are generated from the proposed region-based key agreement algorithm. By using a set comprising an outer group key and subgroup keys a re- gion-based scheme can be efficiently distributed for multiple secure groups. Therefore, the number of rekeying messages, computation and memory can be dramatically reduced. Compared with other schemes, the new proposed region-based scheme can significantly reduce the storage and communication overheads in the rekeying process, with acceptable computational overhead. It is expected that the proposed scheme can be the practical solution for secure group applications, especially for battlefield scenario.

\section{References}

[1] Kefa Rabah, "Theory and Implementation of Elliptic Curve Cryptography," Journal of Applied Sciences, Vol. 5, No. 4, 2005, pp. 604-633.

[2] W. Stallings, "Cryptography and Network Security Principles and Practices," 3rd Edition, Pearson Education.

[3] Y. Wang, B. Ramamurthy and X. K. Zou, "The Performance of Elliptic Curve Based Group Diffie-Hellman Protocols for Secure Group Communication over Ad Hoc Networks," IEEE International Conference on Communication, Vol. 5, 2006, pp. 2243-2248.

[4] Y. Amir, Y. Kim, C. Nita-Rotaru and G. Tsudik, "On the Performance of Group Key-Agreement Protocols," Proceedings of the 22nd IEEE International Conference on Distributed Computing Systems, Viena, Austria, 2002.

[5] M. Steiner, G. Tsudik and M. Waidner, "Key Agreement in Dynamic Peer Groups," IEEE Transaction on Parallel and Distributed Systems, Vol. 11, No. 8, 2000, pp. 769780 . 